\title{
NÖTE UND ZÄSUREN EINES MODERNEN MIGRANTENSCHICKSALS - DER DEUTSCH-POLNISCHE GRENZGÄNGER ARTUR BECKER UND SEIN ROMAN DAS HERZ VON CHOPIN
}

\begin{abstract}
Oppressions and turning points in the fate of the contemporary migrant-Polish-German writer-wanderer Artur Becker and his novel Chopin's Heart

The following article aims at presenting the main emigration-related interpretative aspects of Becker's prose and attempts to put them in the context of the post-colonial theories. The characters created by Artur Becker, who calls himself a "Polish writer of German language", roam continually between the old and the new fatherland and struggle with the feeling of not belonging to any of these worlds. The experience of alienation in foreign country stems from a "transition" in a double sense: on the one side a physical change of the place of residence to Germany which means crossing the "mystical" frontier between the East and the West and on the other a fruitful caesura: the crisis of the identity leads the protagonists of Becker to the conclusion that this hybridity constitutes an intellectual potential and an existential skill.

In the introduction, the foundations of the Postcolonial Theories will be outlined, with the emphasis on the concept of Homi Bhabha which postulates that the fluidity of identity forming in a symbolic "third space", enables the cultural hybridity. Subsequently, after a short presentation of the characters and the work of Artur Becker, the research will focus on the novel Das Herz von Chopin (Chopin's Heart), in which the main character - a financially successful car dealer who emigrated from the communistic Poland to Germany almost 20 years ago while being internally conflicted begins to explore a zone betwixt-and-between to find his own way to happiness.
\end{abstract}

\section{Einführung}

In einem Artikel allen Aspekten der Migrantenexistenz in den Texten von Artur Becker Rechnung zu tragen, wäre unmöglich. In nahezu jedem Roman des polnischen Aussiedlers stellt die Migration vielleicht nicht unbedingt das Hauptthema dar, aber sie macht sicherlich einen essenziellen Anteil daran aus. Die Erfahrung der Fremde wird bei Becker zu einem Übergang in zweierlei Sinn: zum einen als reine Verlegung seines Lebensmittelpunkts, Überbrückung der beinahe mysteriösen Grenze zwischen Ost und West; zum anderen als fruchtbare Zäsur: der Zustand der Identitätskrise mündet in der Einsicht, dass die Hybridität und 
mehrfache Zugehörigkeit als geistiges Potenzial und existenzielle Fähigkeit angesehen werden können.

In der Forschung der so genannten Migrantenliteratur, zu der auch das Schaffen von Artur Becker gezählt wird, werden immer wieder Begriffe verwendet, die durch Postcolonial Studies hervorgebracht wurden. Einer der wichtigsten Theoretiker der postkolonialen Reflexionen war der parsische Literaturwissenschaftler Homi Bhabha. Er entwickelte das Hybriditätskonzept, das den Fundus der Verbindung zwischen Kulturen erfasst: Wechselverhältnisse, Vergleichsversuche, Betonung von Ähnlichkeiten oder Unterschieden. ${ }^{1}$ Mit dem Entwurf der kulturellen Hybridität entwirft Bhabha die Idee des "dritten Raums“ (third space). ${ }^{2}$ Diese spezifische Art der Räumlichkeit bildet sich zwischen den Trägern verschiedener Identitäten heraus, die durch dauerhafte kulturelle Grenzüberschreitungen ständig im Wandel begriffen ist. Derartige Überlappungserscheinungen haben einen enormen Kreativitätsgehalt und provozieren besondere kulturelle Leistungen, wobei das Potenzial der Hybridität gerade in der Migrationsliteratur deutlich wird, denn es sind Migranten - Künstler und Schriftsteller -, die ihre hybride, durch mehrfache Zugehörigkeit gekennzeichnete Identität produktiv ausleben und schöpferisch entfalten können. ${ }^{3}$

\section{Polnischer Autor deutscher Sprache}

Hier und da wird der Vorwurf erhoben, Artur Becker bleibe in seinem Schaffen monothematisch: gescheiterte polnische Grenzgänger suchen ihr Glück im Westen, können aber in ihren Träumen die idyllischen, masurischen Landschaften nicht verlassen. In Das Herz von Chopin erzählt Artur Becker, ein, wie er sich selbst bezeichnet, „polnischer Autor deutscher Sprache“, die Geschichte eines polnischen Migranten, der desillusioniert aus seinem in jener Zeit von Kommunisten regierten Heimatland flieht und beschließt, nach Deutschland zu emigrieren, wo er sich nun seit knapp zwei Jahrzehnten gegen alle Widrigkeiten, die aus dem Leben in der Fremde resultieren, durchsetzt.

Dem Schriftsteller ist das Migrantenschicksal nicht fremd. Geboren wurde er als Artur Bekier am 7. Mai 1968 im polnischen Bartoszyce, wo ihm eine vom Sozialismus und Katholizismus geprägte Kindheit zuteil wurde, auf die er in seinen Werken unaufhörlich zurückgreift. Im Alter von 16 Jahren verlässt er seine masurische Geburtsstadt und seinen Eltern folgend wandert er nach Deutschland aus, wo er bis heute wohnt. Becker wechselt die Sprache und findet im Deutschen seinen literarischen Ton und ein geeignetes Medium, sein Grenzgängertum, das er mit seiner Mig-

\footnotetext{
${ }^{1}$ Vgl. Ekaterina Klüh: Interkulturelle Identitäten im Spiegel der Migrationsliteratur, Würzburg 2009, S. 87.

${ }^{2}$ Vgl. Homi K. Bhabha: Die Verortung der Kultur, Tübingen 2000, S. 126.

${ }^{3}$ Vgl. Klüh: Interkulturelle Identitäten ..., S. 87.
} 
rationssituation eingeleitet hat, künstlerisch zu entfalten. Seit 1997, als sein erster Roman Der Dadajsee erschienen ist, der beinahe sofort nach Erscheinen mit dem Preis des Verbandes Deutscher Schriftsteller in Niedersachsen und Bremen für das beste Buch ausgezeichnet wurde, bringt Becker mit Erfolg Prosatexte und Lyrikbände auf den deutschen Markt. Für das Deutsche als seine Literatursprache, um es mit seinen Worten auszudrücken - seine „Dienstsprache“" ${ }^{\text {, }}$, entschied sich Becker ganz bewusst und publiziert ausschließlich auf Deutsch, also in der Sprache, die er sich nach Ankunft in der Bundesrepublik mühevoll Wort für Wort aneignen musste und in der er seine „sehr strenge Geliebte“ gefunden hat. ${ }^{5}$ Zweifel und Unsicherheiten, vor allem beim Überarbeiten des Textes, lassen ihn aber noch lange nicht los, er nennt es ein „Ringen um die deutsche Sprache“6 ${ }^{\text {“6 }}$ Die Sprache als Ausdruck von Persönlichkeit und Selbstbehauptung spielt eine wichtige Rolle in seinem Werk. Beckers Bücher erfreuen sich großer Begeisterung bei den Lesern und hoher Anerkennung unter den Kritikern, was sich in der Anzahl der ihm zuerkannten Preise und Stipendien widerspiegelt. 2009 wurde Beckers Gesamtwerk mit dem Adelbert-vonChamisso-Preis gewürdigt, der jährlich an Autoren verliehen wird, „,eren Muttersprache und kulturelle Herkunft nicht die deutsche ist, die mit ihrem Werk einen wichtigen Beitrag zur deutschsprachigen Literatur leisten"“7, so die Leitung der Robert Bosch Stiftung, die den Preis seit 1985 vergibt. Beckers Werk als Romancier, Erzähler und Lyriker ,hat nach Ansicht der Jury der deutschen Literatursprache neue Farben und Töne gegeben und die enge Verbundenheit von polnischem und deutschem Kulturraum in poetisch eindrucksvoller Weise bekräftigt. “8 In seinen Büchern schweifen die Geschichten tatsächlich endlos zwischen Polen und Deutschland, zwischen Kindheit und Gegenwart hin und her, sie verflechten und wiederholen sich. Seine Grenzgänger sind Träumer, genauso närrisch wie lebensklug, Wanderer zwischen den Welten, die den Übergang zwischen Ost und West zu überbrücken versuchen, und aufgeweckte Beobachter der deutsch-polnischen Realität.

\section{Die Sogwirkung der polnischen Heimat}

Das Herz von Chopin (2006) reiht sich an vorangegangene und spätere Texte Beckers wie Onkel Jimmy, die Indianer und Ich (2001) oder Wodka und Messer. Das Lied vom Ertrinken (2008) an, denn das psychische und körperliche Grenzgän-

\footnotetext{
${ }^{4}$ Vladimir Balzer: Deutsche Geschichten über die polnische Heimat: Artur Becker, Deutsche Welle, 3. März 2009. Unter http://www.arturbecker.de/Presse/varia/artikel025.html [10.04.2012].

${ }^{5}$ Ebd.

${ }^{6}$ Heide Soltau: Jeder Schriftsteller hat Hausaufgaben: Chamissopreisträger Artur Becker, Deutsche Welle, 27. Dezember 2009. Unter http://www.arturbecker.de/Presse/varia/artikel028.html [10.04.2012].

${ }^{7} \mathrm{http}: / /$ www.bosch-stiftung.de/content/language1/html/4595.asp [20.04.2012].

${ }^{8}$ Ebd.
} 
gertum seiner Haupthelden ist auch hier gut vertreten. Bereits die allerersten Sätze des Romans lassen daran keinen Zweifel:

Jedes Mal, wenn ich beiläufig erwähnte, ich, Chopin, sei ein Soldat der Ewigen Nacht und der Roten Fahne, wurde ich mit Fragen konfrontiert, auf die ich keine Antworten hatte. Ich wollte ja nie so werden wie mein Vater aus Polen. Ich war hier in Bremen nicht aufgewachsen, aber ich saß im ,Piano', meinem Stammcafé, und wartete auf meine Verabredung. ${ }^{9}$

Obwohl die geheimnisvoll klingenden Ausdrücke: Ewige Nacht und Rote Fahne erst im weiteren Verlauf der Geschichte entschlüsselt werden, wird der Umstand der Nichtzugehörigkeit, mit der Chopin offensichtlich nicht ganz zurecht kommt, sofort vom Leser erkannt und begleitet ihn zielgerichtet durch das gesamte Buch.

$\mathrm{Zu}$ seinem Spitznamen kam Chopin als 15-jähriger Rockfan, als ihm nach einer Prügelei die Haare sensationell zu Berge standen. Mitte der 1980er Jahre verlässt er das kommunistische Polen, zieht in den ,goldenen Westen“ und versucht sein Glück als Autohändler in Bremen. Einige deutsche Kritiker sahen in der Figur Chopin nicht nur einen typischen Artur-Becker-Polen, sondern beinahe einen typischen Polen überhaupt ${ }^{10}$; seine Kreation deckt sich in vielen Punkten mit dem stereotypen Bild des polnischen Aussiedlers, der dem Alkohol nicht abgeneigt ist und mit Steuerunterschlagung den deutschen Staat auszutricksen versucht. Doch die Tatsache, dass Becker seinen Protagonisten in den Gebrauchtwagenhandel einsteigen lässt, lässt ein beabsichtigtes Spiel mit nationalen Klischees und Vorurteilen erkennen. Polen als Autodiebe ist wohl das gängigste Stereotyp in der Bundesrepublik über die östlichen Nachbarn, das seinen Ursprung in den frühen 1990er Jahren hat und bis heute in der deutschen Gesellschaft eingebrannt ist. Der Autor setzt stark auf die Zugkraft eingängiger Klischees bei der Gestaltung der Welt seiner Romane, was ihn nach Auffassung vieler Rezensenten zum „Meister der Situationskomik und des bitterbösen Lakonismus “11 macht. In Das Herz von Chopin trifft Ost auf West und diese Begegnung ist befrachtet mit beiderseitigen Vorstellungen, die auf Gegensätzen beruhen: während für den Osten Mythen, Aberglaube, katholische Religion, Spontaneität, Flexibilität und Einfalt, die an Naivität grenzt, stehen, sind Vernunft, Berechenbarkeit und Gewissenhaftigkeit, die glatt in Kaltblütigkeit übergehen kann, Kennzeichen der westlichen Welt. Chopin mit seiner Migrantenexistenz platziert sich genau dazwischen.

Der Migrationsprozess, den Chopin Schritt für Schritt erlebt, wird nur einleitend geschildert und stellt im Grunde kein Hauptthema des Buches dar, obwohl der Migrationshintergrund in Beckers Prosa eine beachtliche Rolle spielt und allen turbulenten

\footnotetext{
${ }^{9}$ Artur Becker: Das Herz von Chopin, München 2008, S. 11. Im weiteren Textverlauf unter der Sigle „HvCh“ und einfacher Seitenzahl.

${ }^{10}$ Gustav Mechlenburg: Akademischer Autohändler. Unter: http://www.arturbecker.de/Presse/herz /rez004.html [27.04.2012].

${ }^{11}$ Sabine Berking: Grenzgänger - Artur Becker erzählt von einem anderen Frédéric Chopin, Frankfurter Allgemeine Zeitung, 13. Oktober 2006. Unter http://www.arturbecker.de/Presse/herz/rez002.html [15.05.2012].
} 
Abenteuern und Wirren einen besonderen, von vielen Kritikern gelobten Charme verleiht. ${ }^{12}$ In der Gegenwart der Romanhandlung hat der Protagonist nicht mehr mit Kulturschock und Einsamkeit zu kämpfen, die vielen der Fremde ausgesetzten Migranten ohne Land- und Sprachkenntnisse zuteilwerden. Vielmehr behauptet Chopin, seine Integration in die deutsche Gesellschaft sei vollkommen abgeschlossen. Er sucht keinen Kontakt zu Polen und zur polnischen Sprache, pflegt keine Jugendfreundschaften mehr und nachdem er vor fast zwanzig Jahren sein Geburtsdorf verlassen hat, besucht er nicht ein einziges Mal die einst geliebten masurischen Landschaften oder gar seine Eltern. Er glaubt B. ein für allemal von seiner geistigen Landkarte getilgt zu haben. Das Heimweh taucht aber unerwartet auf, als eine kleine Notiz zum aktuellen Geschehen, quasi am Rande erwähnt. In seinen Analysen der deutschen Realität zieht er häufig Parallelen zu den polnischen Umständen, wobei jede derartige (meist poetischmelancholische) Rückschau ins Herkunftsland die Ost-West-Unterschiede bloßlegt. Die deutsche Nüchternheit sorgt zwar dafür, dass Chopin lediglich mit einer Prise Anfängerglück ans große Geld kommt, er kann aber seinen finanziellen Erfolg nicht in vollen Zügen genießen. Er fühlt sich niedergedrückt und verstimmt, allerdings ohne dabei explizit seine Fremdheitsgefühle als Gründe hierfür auszudrücken - seine Ankunft in der Bundesrepublik liegt mittlerweile viele Jahre zurück und Chopin, nachdem er einen „Kampf mit der deutschen Sprache“ (HvCh 76) ausfechten musste und trotz aller Widrigkeiten, die ihn im Vergleich mit Gleichaltrigen „um Lichtjahre zurückgeworfen" (HvCh 76) hatten, ist sehr gut in die Aufnahmegesellschaft integriert. Doch die polnische Vergangenheit hinterließ in Chopins Seele wulstige Narben, die ihn keine Ruhe finden lassen. Das Metaphysische und Übersinnliche, die für die osteuropäische Geisteshaltung stehen und die Chopin mit aller Mühe aus seinem Kopf zu verbannen versucht, schleichen sich in seine Träume ein bis sie endlich unerwartet ans Tageslicht kommen und Chopin seine Spagat-Stellung ${ }^{13}$ zwischen zwei Ländern schmerzhaft zu spüren bekommt.

\section{Enklaven, Leitbilder und Symbole}

In beiden Ländern hat Chopin seine zeit- und ortlosen Enklaven, in denen „die Welt abgeschafft" (HvCh 280) ist: in Polen war es ein Hügel am See mit genau

\footnotetext{
${ }^{12}$ Dieser Charme wird auch sprachlich konstruiert: Aufgewachsen im polnischen Kulturraum bringt Becker in seine deutschsprachigen Werke polnische spirituelle Symbolik und surreal anmutende Elemente hinein und dekodieret sie für den deutschen Leser, damit sie weiterhin anziehend exotisch, aber gleichzeitig verständlich sind. Gelegentlich flechtet er in seine Erzählung polnische Begriffe ein. Damit schafft Becker eine Aura der Fremdartigkeit, die dem Romankonzept zugrunde liegt; die Form unterstützt geschickt den Inhalt.

${ }^{13}$ Vgl. Jerzy Kałążny: Schriftsteller im Spagat. Zur deutschsprachigen Gegenwartsliteratur von Autoren polnischer Herkunft, in: Aussiger Beiträge: germanistische Schriftenreihe aus Forschung und Lehre 6, hrsg. von Renata Cornejo, Sławomir Piontek, Sandra Vlasta, Usti nad Labem 2012, S. 119.
} 
zwanzig Pappeln drauf, wo er sich mit seinem Schulfreund Andrzej getroffen hatte; in Bremen ist es eine Dachterrasse, die eine einzigartige Brücke zwischen Bremen und B., zwischen Gegenwart und Vergangenheit und ein Gegenmittel gegen lästige Realität ist:

Sie [die Dachterrasse - Anm. A.K.O.] war der einzige Ort, an dem ich mich entspannen und meinen Job vergessen konnte. Sie lag für mich nicht in Bremen, sondern in Masuren. Am Dadajsee. Ich bildete mit ein, dort gäbe es keine Aufseher und Wächter und Steuereintreiber. Keine Kontrolleure, Kunden und Verkäufer. Der Dadajsee befand sich in einem anderen Raum, in einer anderen Zeit, nicht in Polen, Masuren, im Ermland oder auf der Erde. Ohne diese Zuflucht hätte ich mein Leben in Deutschland schwer ertragen können. (HvCh 188)

Es sind nahezu heilige Orte, die Chopin vor fremden Blicken schützen will; in B. befand sich dort eine „Kommandozentrale“, wo er als Junge mit seinem Freund philosophierte, träumte und auf die Liebe wartete. Auf der Dachterrasse in Bremen genießt er einerseits seine Einsamkeit und lässt seine Gedanken ungehemmt fließen, andererseits will er sein geheimes Versteck mit seiner Geliebten teilen, die sich von diesem Refugium genauso begeistert zeigt: „Ich fühle mich auf deinem Dach, als wären wir gar nicht in Deutschland! Lass uns hier ein Haus bauen!“ (HvCh 128) Mit M.M. auf seiner Dachterrasse fühlt sich Chopin auf eine besondere Art und Weise glücklich, fern vom Stigma des Migranten, des Nichtdazugehörenden. Diese Glücksgefühle verbindet er mit dem, was er glaubte, wie eine lästige Bürde hinter sich gelassen zu haben: mit Polen.

Mit M.M. fühlte ich mich endlich nicht mehr wie ein Sonderling, als einer, der ständig aufklären musste, was ihm fehlte und dass ihm der Fuß oder sonst was wehtat. Ich konnte meine Musik auflegen und so sein wie früher. Als wäre ich wieder mit Andrzej zusammen [...]. Als hätte ich Jolka wiedergetroffen. (HvCh 128)

Chopin nähert sich nach und nach der Einsicht, dass das Glück, das einzige erstrebenswerte Ziel im Leben, sich nicht durch das Land, im dem man wohnt, definiert.

Der Weg, den Chopin zurücklegt, mag zwar in seinen Augen gekrümmt erscheinen, erweist sich im Laufe der Geschichte aber als eine deutliche Entwicklungslinie. Dies gilt insbesondere in Hinsicht auf Chopins erträumte und tatsächlich ausgeübte Berufe bzw. Arbeitsfelder und bewohnte Orte, in der die Alliteration sichtbar wird. Als Junge aus B. schmiedete Chopin Pläne, Polizeikommissar zu werden, wie sein Vorbild, der Schauspieler Alain Delon, den er für seine Rolle in dem Thriller Der eiskalte Engel bewundert hat. Angekommen in Bremen fängt er ein Kriminalistik-Studium an, bricht es aber bald ab, zugunsten der lukrativen Tätigkeit als Autohändler. Nach all dem turbulenten Wirbel in Bremen entscheidet sich Chopin schließlich, die Führung eines Antiquitätenladens in Berlin zu übernehmen. Diese sprachlich durch Alliteration akzentuierten Konstel- 
lationen: B. - Bremen - Berlin und Alain Delon - Autohändler - und Antiquar betonen Kontinuität und Zweckmäßigkeit aller von Chopin unternommen Schritte. ${ }^{14}$ Seine letzte im Roman eingeleitete Etappe verspricht Stabilität: zum einen kann sich der abgekämpfte Grenzgänger in Berlin, der Stadt aller Nationalitäten, wo in der Vielzahl von Sprachen und Kulturen die nationale Zugehörigkeit im herkömmlichen Sinne an Bedeutung verliert, zum anderen im Antiquitätenladen, der Vergangenheit und Gegenwart in sich vereint, erst einmal Ruhe gönnen. Chopin steht somit vor der Aussicht, sich von der Sogwirkung seiner polnischen Heimat zu emanzipieren, ohne sie aber zu verleugnen, und sein Glück jenseits der Nationalitätsfrage zu finden. Doch Artur Becker lässt seinen Roman mit Chopins Feststellung „Unser Kampf ist verloren“ enden. Nach allen Krisen und Abenteuern muss er feststellen, dass er seine Spagat-Stellung ${ }^{15}$ zwischen zwei Ländern, die seit der Ankunft in der Bundesrepublik mehr oder weniger lästig für ihn ist, wahrscheinlich nie überwinden wird. Betrachtet man den Schelmenroman als eine Auseinandersetzung mit der polnischen Identität, so bekommt der Titel eine tiefere Bedeutung: der namensgebende Klaviervirtuose war selbst ein Emigrant und verfügte kurz vor seinem Tod, dass seine Gebeine zwar in Paris bestattet werden sollten - sein Herz aber in einer Warschauer Kirche. ${ }^{16}$ Chopin lebt zwar in der Fremde, der er seinen wirtschaftlichen Erfolg verdankt, doch sein Herz, oder wenigstens ein Stück davon, gehört immer noch nach Polen.

\section{Migration als ,eine universelle Form zu existieren“617}

Kuba, der Protagonist des 2008 veröffentlichten Romans Wodka und Messer. Lied vom Ertrinken kommt zu dem Schluss:

Die Emigration ist eine Fünfstufenrakete. Eins - man flieht; zwei - man gewöhnt sich; drei - man vergisst; vier - man erinnert sich; und fünf - man will zurückkehren, aber es geht nicht mehr. ${ }^{18}$

Chopin erlebt seine existenzielle Krise als Migrant zwischen der dritten und vierten Stufe - der Ursprung vieler seiner Probleme platziert sich zwischen dem Vergessen bzw. Vergessen-Wollen und der Erinnerung, die häufig am Rande des

\footnotetext{
${ }^{14}$ Vgl. Sławomir Piontek: Zwischen B und B. Identitätsräume bei Artur Becker, in: Aussiger Beiträge: germanistische Schriftenreihe aus Forschung und Lehre 6, hrsg. von Renata Cornejo, Sławomir Piontek, Sandra Vlasta, Usti nad Labem 2012, S. 141.

${ }^{15}$ Vgl. Kałążny: Schriftsteller im Spagat.

${ }^{16}$ Chopins Biographen geben allerdings an, der polnische Komponist hätte es lediglich aus Angst davor, lebendig begraben zu werden, angeordnet.

${ }^{17}$ Ingrid Reichel: Kraft der Erinnerung aus der Kindheit. etcetera 35, Heftthema: KIND, März 2009. Unter: http://www.arturbecker.de/Presse/varia/artikel022.html [10.04.2012].

${ }^{18}$ Artur Becker: Wodka und Messer: Lied vom Ertrinken, Frankfurt a.M. 2008, S. 454.
} 
Bewusstseins wahrgenommen wird. Auf dieser Achse kommt es zu Reibungen: auch viele Jahre seit seiner Ankunft in der Bundesrepublik fühlt sich Chopin andersartig er hat keinen Bezug mehr zu seinem Heimatland Polen, eine Rückkehr zieht er nicht einmal in Betracht, empfindet sich aber trotz seiner weitgehenden Eingliederung in die deutsche Gesellschaft ebenso wenig als Deutscher. Als Chopin von seiner Freundin verlassen und von den Geschäftspartnern betrogen wurde, kommt es zu einem dramatischen Umbruch, der sich doch als fruchtbare Zäsur erweist: Beckers Protagonist bekommt die Möglichkeit, seinen „dritten Raum“, „die Migrantenkultur der Zwischenzone ${ }^{\text {“19 }}$ im Sinne Homi Bhabhas aufzubauen und das Beste aus seiner Migrationssituation zu machen. Die postkoloniale Theorie legt verschiedene Mittel der Auseinandersetzung mit Nationalitätsfragen, Zugehörigkeit und Fremdbestimmung dar. Mit Zuschreibungen durch die Außenwelt bzw. die Aufnahmegesellschaft, welche die in Deutschland lebenden Migranten häufig zu verwinden haben, beschäftigt sich Artur Becker nur indirekt; vielmehr reflektiert er die Grenzziehungen, die vor allem im Kopf seines Helden selbst existent sind. Der Erfolg oder Misserfolg eines selbstbestimmten Handels ist größtenteils von seiner Fähigkeit abhängig, sich über nationale Kategorien hinweg persönlich weiterzuentwickeln und die Selbsterkundung nach eigenen Definitionsmustern zu vollziehen.

Der Autor selbst geht auf Distanz zu nationalen Befindlichkeiten und der so genannten osteuropäischen Seele, und streitet es ab, diese in seinen Romanen ergründen zu wollen. Trotzdem nennt er die Emigration „ein Glücksgefühl“ und „eine universelle Form zu existieren ${ }^{\text {‘20 }}$, da erst die Migrationssituation und der Zusammenprall der Lebensstile die Beobachtungsgabe schärfen und einen wachen Blick für beide Realitäten bewahren lassen: einerseits für das Geburtsland, da man zwangsweise den direkten Bezug zum zeitnahen politischen und gesellschaftlichen Geschehen verliert, andererseits für den Alltag der Wahlheimat, in der aus der Vogelperspektive betrachtet, vieles Gewohnte, wenn nicht absurd, so doch höchst befremdlich erscheint. Becker gibt an, seine literarischen Untersuchungen sollten vor allem einen Hauptgedanken in den Vordergrund rücken, nämlich dass wir in erster Linie keine Vertreter der jeweiligen Nationalitäten, sondern einfach nur Menschen sind. Er führt dabei ein Beispiel aus dem Hinduismus an:

[...] Krishna Arjuna erklärt: »Du bist doch kein Hund. Wenn man dich fragt, wer du bist, darfst du nicht wie ein Hund antworten und bellen. Du bist doch in erster Linie ein kosmisches Wesen und ein Mensch.« Das symbolische Bellen eines Hundes ist das, was in unserem Reisepass steht. Es besagt jedoch gar nichts über uns und wie wir existieren. Emigration ist, wenn man sie nützen kann, eine gute Form, um zu einem kosmischen oder eben universellen Menschen zu werden. Also zu einem »Erdling «. ${ }^{21}$

\footnotetext{
${ }^{19}$ Bhabha: Die Verortung der Kultur, S. 335.

${ }^{20}$ Ingrid Reichel: Kraft der Erinnerung aus der Kindheit. etcetera 35, Heftthema: KIND, März 2009. Unter: http://www.arturbecker.de/Presse/varia/artikel022.html [10.04.2012].

${ }^{21} \mathrm{Ebd}$
} 
$\mathrm{Zu}$ dieser Anschauung gelangt Chopin nach der Generalbeichte nach dem Bruch mit seiner Geliebten, als er verwirrt in einem unbekannten Raum aufwacht und dort zufällig einen menschlichen Totenschädel auffindet, der ihn auf diesen Gedanken bringt:

Vielleicht würde mir nach meinem Tod ja etwas Ähnliches zustoßen: Irgendjemand könnte meine Knochen ausgraben und in seiner Bibliothek ausstellen wie in einem Museum. Für eine Ausstellung wurden wir aber nicht geschaffen. Wir Menschen mussten mehr sein. Wir mussten erstrahlen und brennen. Und vor allen Dingen sein. (Hervorhebung vom Autor, HvCh 246)

Im Kontext der fortschreitenden Globalisierungs- und Hybridisierungsprozesse, die in der Überlappung unterschiedlicher Kulturelemente den „Dritten Raum“ entstehen lassen, ist diese Feststellung von besonderer Bedeutung. In einer Welt, die unaufhaltsam zu einer globalen Stadt zusammenwächst, in der die Entfernungen dahinschmelzen und nationale Grenzen verschwimmen, sind Kulturtransfer und Migration immer mehr zu Schlüsselbegriffen geworden.

Mittlerweile ist das Leben zwischen zwei Sprachen kein individuelles Phänomen mehr: die Erfahrungen der Fremde und des Fremdseins gehören zu den Grundzügen der Postmoderne. Wie das Beispiel Chopins deutlich macht, stiftet die Migrationssituation keine Identität - wenigstens keine nationale. Sie stigmatisiert nicht mehr und verbunden mit der erfolgreichen Integration und gesellschaftlichen Teilhabe, die im breiteren Kontext der sozialen Vitalität und dem kulturellen Reichtum des Aufnahmelandes zugrunde liegen, ermöglicht es einen tieferen Einblick in beide Kulturen, ihre Politik, Geschichte und Ideologien.

Saša Stanišić, ein aus Bosnien und Herzegowina stammender deutschsprachiger Schriftsteller, berichtet in einem Aufsatz: „Wie ihr uns seht. Über drei Mythen vom Schreiben der Migranten" von seinem Treffen mit Artur Becker:

$\operatorname{Er}$ [Artur Becker - A.K.O.] erzählte mir, er würde immer einen einzigen ästhetischen und metaphysischen Topos umkreisen: Geschichten, die zwischen zwei Kulturen spielen. Man könnte sein CEuvre als Literatur kultureller Synthese bezeichnen. ${ }^{22}$

Becker wertet in jedem folgenden Roman von neuem seine Exilgeschichte aus, sucht nach einer neuen Identität und Identifikation, beobachtet scharfsinnig die Realität seiner Wahlheimat, ohne aber dabei allezeit das Neue an ihr zu betonen oder die Qual eines der Fremde ausgesetzten Migranten zu behandeln. Trotzdem verzichtet er nicht auf das exotische Flair, das er seiner osteuropäischen Herkunft verdankt, und versucht mit seiner Literatur auf eine kulturelle Synthese hinzuarbeiten, die ebenso utopisch wie erstrebenswert ist.

${ }^{22}$ Saša Stanišić: Wie ihr uns seht. Über drei Mythen vom Schreiben der Migranten, in: Eingezogen in die Sprache, angekommen in der Literatur, hrsg. von Uwe Pörksen, Bernd Busch, Valerino 2008, S. 107. 


\section{Literatur}

Balzer, Vladimir: Deutsche Geschichten über die polnische Heimat: Artur Becker, Deutsche Welle, 3. März 2009. Unter http://www.arturbecker.de/Presse/varia/artike1025.html [10.04.2012].

Becker, Artur: Das Herz von Chopin, München 2008.

Becker, Artur: Wodka und Messer: Lied vom Ertrinken, Frankfurt a.M. 2008.

Berking, Sabine. Grenzgänger - Artur Becker erzählt von einem anderen Frédéric Chopin, Frankfurter Allgemeine Zeitung, 13. Oktober 2006. Unter http://www.arturbecker.de/Presse/herz/rez002.html [15.05.2012].

Bhabha, Homi K.: Die Verortung der Kultur, Tübingen 2000.

Klüh, Ekaterina: Interkulturelle Identitäten im Spiegel der Migrationsliteratur, Würzburg 2009.

Kałążny, Jerzy: Schriftsteller im Spagat. Zur deutschsprachigen Gegenwartsliteratur von Autoren polnischer Herkunft, in: Aussiger Beiträge: germanistische Schriftenreihe aus Forschung und Lehre 6, hrsg. von Renata Cornejo, Sławomir Piontek, Sandra Vlasta, Usti nad Labem 2012, S. 119-131.

Mechlenburg, Gustav: Akademischer Autohändler. Unter: http://www.arturbecker.de/Presse/herz/rez 004.html [27.04.2012].

Piontek, Sławomir: Zwischen B und B. Identitätsräume bei Artur Becker, in: Aussiger Beiträge: germanistische Schriftenreihe aus Forschung und Lehre 6, hrsg. von Renata Cornejo, Sławomir Piontek, Sandra Vlasta, Usti nad Labem 2012, S. 133-142.

Reichel, Ingrid: Kraft der Erinnerung aus der Kindheit. etcetera 35, Heftthema: KIND, März 2009. Unter: http://www.arturbecker.de/Presse/varia/artikel022.html [10.04.2012].

Soltau, Heide: Jeder Schriftsteller hat Hausaufgaben: Chamissopreisträger Artur Becker, Deutsche Welle, 27. Dezember 2009. Unter http://www.arturbecker.de/Presse/varia/artikel028.html [10.04.2012].

Stanišić, Saša: Wie ihr uns seht. Über drei Mythen vom Schreiben der Migranten, in: Eingezogen in die Sprache, angekommen in der Literatur, hrsg. von Uwe Pörksen, Bernd Busch, Valerino 2008. 